\title{
Investigation of the Relationship Between Wrist Ganglion Cysts and the Ulnar Variance Using 3-Tesla Magnetic Resonance Imaging
}

\author{
(1) Murat Bayav \\ Eskisehir City Hospital, Clinic of Radiology, Eskisehir, Turkey
}

\section{Abstract}

Aim: A ganglion cyst is the most common soft tissue mass in the wrist and although the etiology of ganglion cysts remains unclear, the commonly accepted theory is that they are due to acute or chronic stress in the joint, which may be also caused by ulnar variance. The study aims to evaluate the relationship of ulnar variance in the wrist with ganglion cysts in this region using magnetic resonance imaging (MRI).

Methods: In this cross-sectional study, patients aged 18 years and over who underwent wrist MRI between January 1, 2019 and December 31, 2019 were evaluated. The MR images and patient demographic data were obtained from a hospital database. The presence and size of the ganglion cyst and the amount of ulnar variance were assessed using the MR images. The ulnar variance was classified into negative, positive, and neutral groups to compare ganglion cyst presence between these groups.

Results: A total of 216 wrists from 206 patients were included in the study. The ulnar variance was negative in 100 (46.3\%) wrists, neutral in 99 (45.8\%), and positive in 17 (7.9\%). One or more ganglion cysts were observed in 130 (60.2\%) wrists, and no ganglion cyst was found in 86 (39.8\%). Compared to the neutral group, the patients with negative ulnar variance had a six-fold increased risk, and those with positive ulnar variance had a 12 -fold increased risk of having a ganglion cyst.

Conclusion: The presence of positive or negative ulnar variance increases the risk of having a ganglion cyst in the wrist. This may be related to soft tissue trauma caused by joint instability due to ulnar variance. MRI can be performed to evaluate the presence of ganglion cysts in patients with positive or negative ulnar variance detected on direct radiography.

Keywords: Wrist joint, ganglion cysts, ulna, magnetic resonance imaging

\section{Introduction}

Ulnar variance is the relative length of the distal articular surface of the ulna to the radius (1). Ulnar variance measurement is generally performed in the neutral position using postero-anterior radiographs. However, the soft tissue resolution of radiographs is low. A recent study shows that ulnar variance on wrist magnetic resonance imaging (MRI) can be measured reliably (2). Another recent study shows positive ulnar variance increases pathologies of the triangular fibrocartilage complex (TFCC) and longitudinal carpal instability using MRI (3).

A ganglion cyst is the most common benign soft tissue mass in the wrist. It is diagnosed based on imaging with sonography or MRI (4). Although the etiology of ganglion cysts remains unclear, the commonly accepted theory is that due to acute or chronic stress in the joint, the fluid in the joint leaks between the tissues, creating an inflammatory reaction (5). It is known that both negative and positive ulnar variances cause carpal instability (1). However, there is not sufficient information in the literature concerning the relationship between ulnar variance and ganglion cysts located in the wrist.

This study aimed to evaluate the relationship between ulnar variance in the wrist and ganglion cysts in this region using 3-Tesla MRI. 


\section{Methods}

\section{Ethical Statement}

An ethics committee and institution approval were obtained before starting the study (approved by the ethics committee of Eskisehir Osmangazi University, approval number: 2020/541, date: 12.01.2021). Informed consent was not required by the ethics committee due to the retrospective nature of the study.

\section{Patients}

In this retrospective cross-sectional study, patients aged 18 years and over who underwent wrist MRI between January 1, 2019 and December 31, 2019 were retrospectively evaluated. Broad inclusion criteria which cover most of the patients without considering clinical symptoms were used in order to investigate the relationship between ulnar variance and the presence of ganglion cysts in many cases independent of clinical symptoms. The exclusion criteria were MRI images with insufficient diagnostic quality, those that contained congenital or sequelae changes that would disrupt the wrist bone alignment, which makes it impossible to measure ulnar variance, and those that were not obtained in the neutral position. We identified a total of 216 wrist images of 206 patients meeting the study criteria.

\section{Imaging Protocol and Image Analysis}

Images were obtained using the Siemens Magnetom Skyra $^{\mathrm{TM}}$ (Siemens AG, Muenchen, Germany) 3-Tesla MRI device. The imaging protocol was as follows: axial fat suppressed (FS) turbo spin echo (TSE) proton densityweighted imaging (PD-WI) (TR, 4860 ms; TE, 46 ms; NEX, 1; ETL, 7; section thickness, $2.5 \mathrm{~mm}$; interslice gap, 0.25 $\mathrm{mm}$; flip angle, $150^{\circ}$; matrix size, $320 \times 320$; and field of view, 100x100 mm), coronal FS TSE PD-WI (TR, $2340 \mathrm{ms;}$ $\mathrm{TE}, 37 \mathrm{~ms}$; NEX, 1; section thickness, $3 \mathrm{~mm}$; interslice gap, $0.3 \mathrm{~mm}$; flip angle, $150^{\circ}$; matrix size, 320x320; and field of view, 100×100 mm), coronal TSE T1 WI (TR, 2340 $\mathrm{ms}$; TE, $37 \mathrm{~ms}$; ETL, 9; NEX, 1; section thickness, $3 \mathrm{~mm}$; interslice gap, $0.3 \mathrm{~mm}$; matrix size, 320x320; and field of view, 100x100 mm), 3D TSE T2 WI (TR, $17 \mathrm{~ms} ;$ TE, 5,67 $\mathrm{ms}$; ETL, 2; NEX, 1; section thickness, $0.4 \mathrm{~mm}$; matrix size, $320 \times 320$; and field of view, $100 \times 100 \mathrm{~mm}$ ), and sagittal FS TSE T2 WI (TR, 3800 ms; TE, 78 ms; NEX, 1; ETL, 9; section thickness, $2.5 \mathrm{~mm}$; interslice gap, $0.25 \mathrm{~mm}$; flip angle, $150^{\circ}$; matrix size, $288 \times 384$; and field of view, $90 \times 120 \mathrm{~mm}$ ).

The images were evaluated by a radiologist with two years of experience in MRI. The presence of ganglion cysts was evaluated from coronal T2 WI and T1 WI together with FS PD-WI in the axial and coronal planes. Ganglion cysts were defined as lesions with low T1 signal and high T2 and PD signals similar to synovial fluid, with a well- demarcated nodular appearance, adjacent to the joint space or tendons (Figure 1). For the patients detected to have ganglion cysts, the size measurement of the cysts was performed using 3D T2 WI due to the thinner section thickness. The longest diameter of the ganglion cyst was accepted as the size. The ulnar variance was evaluated using coronal T1 WI. In this evaluation, the distance between the transverse plane passing through the lunate fossa of the radius and the transverse plane passing through the ulnar head was measured (Figure 2). Positive or negative ulnar variance amounts below 1 $\mathrm{mm}$ were considered neutral. A dataset was created by recording the demographic data of the patients with the measurement data obtained.

\section{Statistical Analysis}

Statistical analysis was performed using the IBM SPSS version 25 (IBM Corp., Armonk, New York, NY, USA) software package. Mean standard deviation (minimummaximum) values were used to represent continuous data with a normally distributed distribution, whereas frequency and percentage were used to represent categorical data. The correlation analysis of the amount of ulnar variance and the diameter of the ganglion cysts was performed with the Pearson correlation test. Ulnar variance groups were compared using the chi-square test in terms of categorical data such as the presence of ganglion cysts, while the independent samples t-test was used to compare the mean values of continuous data such as the ganglion cyst diameter between the groups. In addition, for the groups with positive and negative ulnar variances, the odds ratio (OR) for having a ganglion cyst was calculated in comparison with the ulnar neutral variance group. A p-value of 0.05 was considered statistically significant.

\section{Results}

Of the 206 patients included in the study, 93 (45.1\%) were male and 113 (54.9\%) were female. The mean age of the patients was $35.03 \pm 12.51$ (18-74) years. Of the 216 wrists imaged, 116 (53.7\%) belonged to the right wrist and $100(46.3 \%)$ to the left wrist. The most common reasons for imaging indications included trauma in 65 (30.1\%) patients, tenosynovitis in $42(19.4 \%)$, pain in 41 $(19 \%)$, and ganglion cysts and soft tissue masses in 41 (19\%). Ulnar variance was negative in 100 (46.3\%) wrists, neutral in $99(45.8 \%)$, and positive in $17(7.9 \%)$. The mean amount of ulnar variance was $3.15 \pm 1.38(1.10-9.18) \mathrm{mm}$ for the wrists with negative ulnar variance and $2.02 \pm 0.67$ (1.16-3.66) $\mathrm{mm}$ for those with positive ulnar variance. One or more ganglion cysts were observed in 130 (60.2\%) wrists, and no ganglion cyst was found in 86 (39.8\%) (Table 1). Among the wrists with ganglion cysts, there was one ganglion cyst in 111 (85.4\%), two in 16 (12.3\%), and 

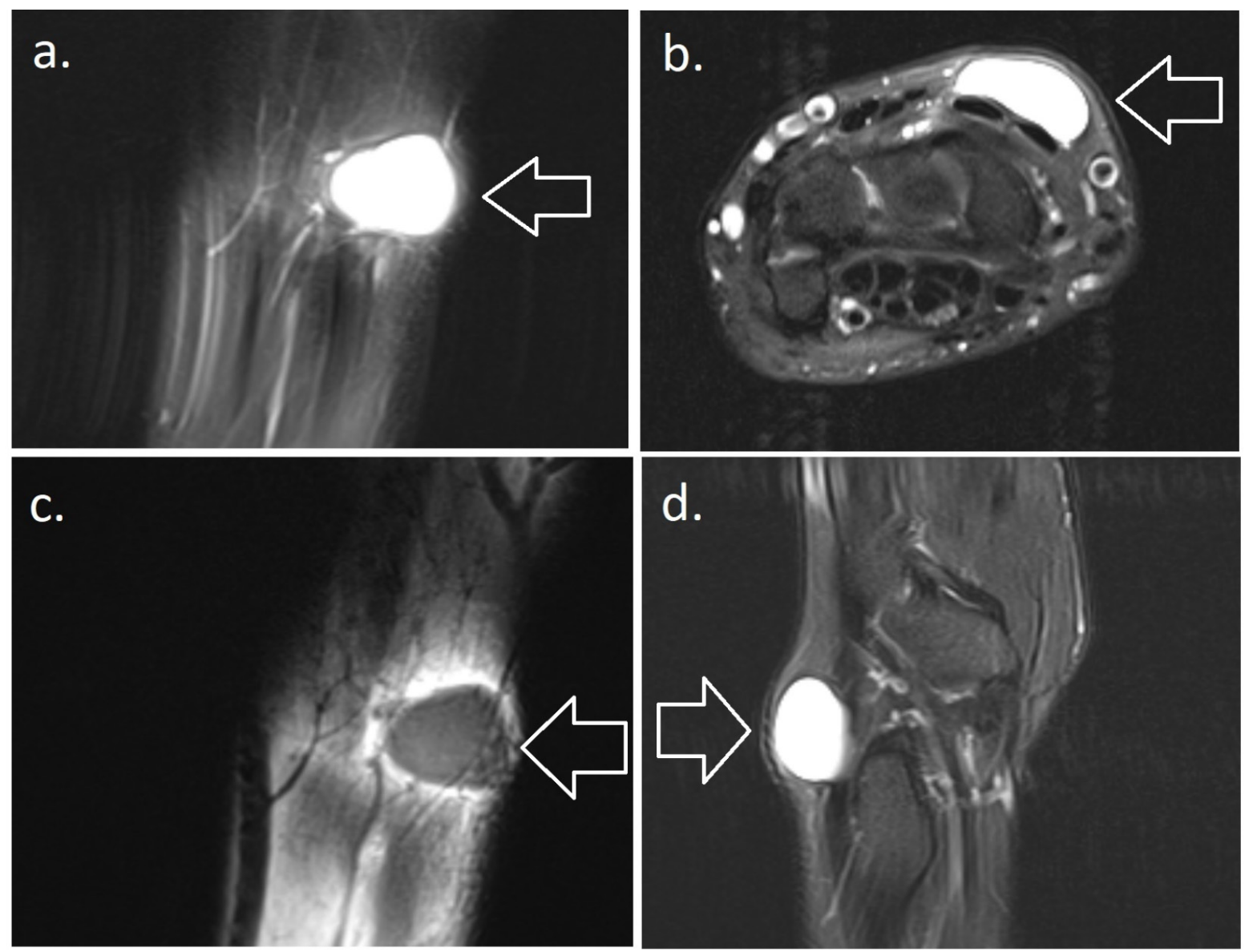

Figure 1. a. Coronal fat-suppressed proton density-weighted and b. Axial fat-suppressed proton density-weighted images showing a well-demarcated hyperintense ganglion cyst adjacent to the extensor tendons. c. Coronal T1 and d. sagittal FS T2-weighted images of the same cyst (white arrows)

three or more in three (2.3\%). The female had a ganglion cyst presence ratio of $63.6 \%$, while the male had a ratio of $55.8 \%$. Considering the largest cyst of the cases with more than one ganglion cyst, the location of the cyst was in the palmar area in 70 (53.8\%) cases, dorsal in 43 (33.1\%), and the interosseous area in 17 (13.1\%).

\begin{tabular}{|l|l|l|}
\hline Table 1. Patient characteristics \\
\hline Mean age, years & $35.03 \pm 12.51(18-74)$ \\
\hline \multirow{2}{*}{ Gender } & Male & $93(45.1 \%)$ \\
\cline { 2 - 3 } & Female & $113(54.9 \%)$ \\
\hline \multirow{2}{*}{ Wrist side } & Right & $116(53.7 \%)$ \\
\cline { 2 - 3 } & Left & $100(46.3 \%)$ \\
\hline \multirow{2}{*}{ Ganglion cyst } & Present & $130(60.2 \%)$ \\
\hline \multirow{4}{*}{ Ulnar variance } & Absent & $86(39.8 \%)$ \\
\hline \multirow{2}{*}{$\begin{array}{l}\text { Data are shown as mean } \pm \text { standard deviation (min-max) and } \mathrm{n}(\%) \\
\text { Min: Minimum, max: Maximum }\end{array}$} & Negative & $100(46.3 \%)$ \\
\cline { 2 - 3 } & Neutral & $99(45.8 \%)$ \\
\hline
\end{tabular}

For the patients with ganglion cysts, the mean cyst diameter was $9.04 \pm 4.55(3.3-25.82) \mathrm{mm}$ in those with negative ulnar variance, $8.45 \pm 4.11(2.8-20.44) \mathrm{mm}$ in

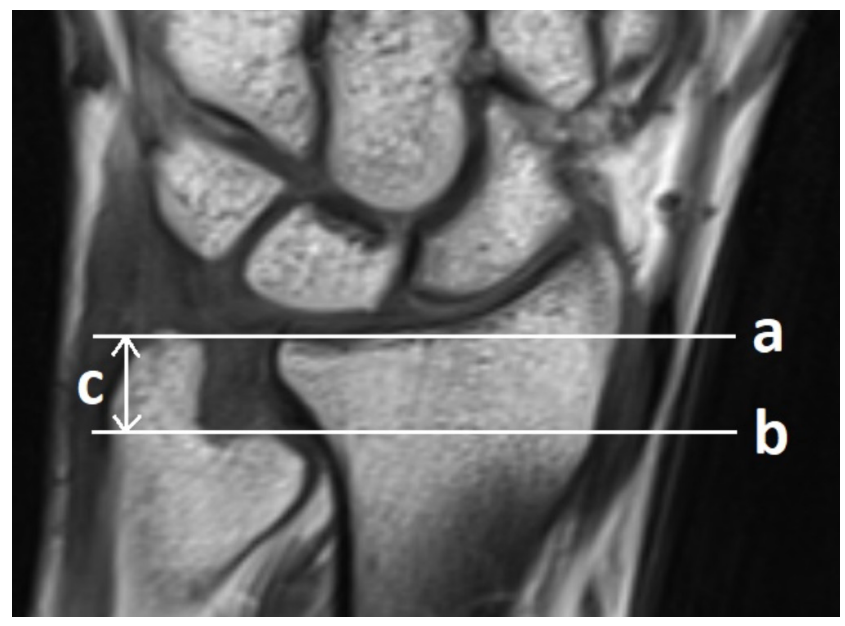

Figure 2. Coronal T1-weighted image. a. Transverse plane in the lunate fossa of the radius, b. transverse plane passing through the distal ulnar head, and $\mathbf{c}$. ulnar variance amount 
those with neutral ulnar variance, and 9.77 \pm 5.01 (3.5920.89) $\mathrm{mm}$ for those with positive ulnar variance, indicating no statistically significant difference between the negative and positive groups and the neutral group ( $p=0.49$ and $p=0.90$, respectively). When the ganglion cyst presence according to ulnar variance was examined, compared to the neutral group, those with negative ulnar variance had a six-fold increased risk [OR: 5.95; 95\% confidence interval $(\mathrm{Cl})$ : 3.18-11.11] and those with positive ulnar variance had a 12-fold increased risk of having a ganglion cyst (OR: $12.56 ; 95 \% \mathrm{Cl}: 2.72-58.07)$ of having a ganglion cyst. Ganglion cysts were statistically significantly more common among the patients with negative or positive ulnar variance (Table 2) (Pearson's $\chi^{2}: 40,325, p<0.001$ ).

\section{Discussion}

At the end of the study, the risk of having a ganglion cyst was found to be approximately six times greater in the group with negative ulnar variance and 12 times greater in the group with positive ulnar variance compared to the neutral ulnar variance. This reveals that having a ganglion cyst is particularly closely related to having positive ulnar variance. We have not encountered any publication in the literature that demonstrates this relationship. However, Turan et al. (6) showed a relationship between negative ulnar variance and scaphoid bone fractures. In addition, Yoshioka et al. (7) reported that ulnar variance was positively correlated with the TFCC angle and negatively correlated with its thickness. In another study, Roh et al. (8) determined that positive ulnar variance was more common in patients with a symptomatic TFCC tear. These studies reveal the effect of wrist instability caused by positive or negative ulnar variance on bone and soft tissues. Also, shortening of the ulna in positive ulnar variance patients by osteotomy resulted in improved carpal stability outcomes (9). In the etiology of ganglion cysts, there is acute or chronic stress-induced leakage of the joint fluid, which results in an inflammatory reaction as discussed in the introduction (5). This also explains the effect of positive and negative ulnar variance on ganglion cysts observed in our study.
Ghalimah et al. (10) found the negative ulnar variance rate to be $56.2 \%$ and the neutral variance rate to be $43.8 \%$ in X-ray (plain radiography) examinations performed on the wrist in healthy volunteers, and they did not find any positive ulnar variance. In our study, although the rate of neutral variance was similar, a positive variance was observed at a rate of approximately $8 \%$, and the negative variance rate was $46.3 \%$. This may be associated with the symptomatic nature of the cases with positive variance since we did not exclude patients showing symptoms from our sample. In another study conducted with X-ray examinations, it was revealed that there was excellent agreement between the observers and the maximum inter-observer difference was $1 \mathrm{~mm}$ in the assessment of ulnar variance (11). Studies demonstrate the success of $X$-ray imaging in evaluating ulnar variance. However, the soft tissue resolution of $X$-ray imaging is low. Due to the low soft tissue resolution of X-ray, Yoshioka et al. (7) chose to use high-resolution gradient echo T2 ${ }^{*} \mathrm{MRI}$ images obtained with a 1.5-Tesla device, and Roh et al. (8) used conventional T1 and T2 WI obtained with a 3-Tesla device to evaluate ulnar variance. In the current study, we also obtained images using a 3-Tesla MRI device to evaluate ganglion cysts and used conventional T1 WI to evaluate ulnar variance.

Zhang et al. (12), who evaluated ganglion cysts with sonography, stated that $69 \%$ of the ganglion cysts associated with the wrist were seen on the palmar surface and $31 \%$ on the dorsal face. In an MRI study, Lowden et al. (13), investigating the presence of ganglion cysts in a healthy volunteer population, reported that $86 \%$ of the ganglion cysts originated from the palmar surface, mostly from the opening between the radioscaphocapitate and the long radiolunate ligament. In addition, they found the frequency of ganglion cysts among healthy volunteers to be $51 \%$ (13). Similarly, in the current study, although ganglion cysts were mostly seen on the palmar side, the incidence of ganglion cysts was found at a rate of approximately $60 \%$, and this was attributed to the inclusion of symptomatic patients in the sample.

In a study examining the surgical results of ganglion cysts, the recurrence rate of ganglion cysts was found

\begin{tabular}{|c|c|c|c|c|}
\hline \multicolumn{5}{|c|}{ Ganglion cyst } \\
\hline Ulnar variance & $\begin{array}{l}\text { Negative } \\
\text { Neutral } \\
\text { Positive } \\
\text { Total }\end{array}$ & $\begin{array}{l}\text { Absent } \\
22(10.2 \%) \\
62(28.7 \%) \\
2(0.9 \%) \\
86(39.8 \%)\end{array}$ & \begin{tabular}{|l} 
Present \\
$78(36.1 \%)$ \\
$37(17.1 \%)$ \\
$15(6.9 \%)$ \\
$130(60.2 \%)$
\end{tabular} & $\begin{array}{l}\text { Pearson's } \chi 2: 40,325 \\
(p<0.001)\end{array}$ \\
\hline
\end{tabular}


to be approximately $10 \%$, and no significant relationship was detected between recurrence and gender, age, wrist side, or cyst localization (14). Ganglion cysts can also be resected arthroscopically with a low rate of complications and recurrence when they are symptomatic (15). Although ganglion cysts have been mostly evaluated surgically in the literature, in an MRI study of wrist ganglion cysts in pediatric cases, Bracken and Bartlett (16) found accompanying imaging findings in those with ganglion cysts, listing them as joint effusion, trauma-related bone marrow edema and fractures, TFCC tears, and negative ulnar variance, in order of frequency. We did not find any study in the literature that directly investigated the relationship between ulnar variance and ganglion cysts.

\section{Study Limitations}

The main limitations of the study are the absence of imaging and clinical follow-up of the patients due to the retrospective design. In addition, the results cannot be generalized to the whole population since the study was conducted in a single center. Lastly, only radiological findings were evaluated, and clinical symptoms or surgical findings were not included in the study. On the other hand, this study was the first one evaluating the relationship of ulnar variance in the wrist with ganglion cysts, and further studies that include clinical features of ganglion cysts can provide further information on the relationship.

\section{Conclusion}

Ulnar variance can be easily evaluated with direct radiography performed in the first line of imaging undertaken due to wrist pain. As shown in our study, the risk of having ganglion cysts in patients with positive or negative ulnar variance is considerably higher compared to patients with neutral ulnar variance. In this situation, MRI will be useful in revealing accompanying ganglion cysts and other soft tissue disorders among patients with negative or positive ulnar variance.

\section{Ethics}

Ethics Committee Approval: The study was approved by the ethics committee of Eskisehir Osmangazi University with approval number: 2020/541, date: 12.01.2021.

Informed Consent: Informed consent was not required by the ethics committee due to the retrospective nature of the study.

Financial Disclosure: The author declared that this study received no financial support.

\section{References}

1. Cerezal L, del Piñal F, Abascal F, García-Valtuille R, Pereda T, Canga A. Imaging findings in ulnar-sided wrist impaction syndromes. Radiographics 2002;22:105-21.
2. Serfaty A, Costa HP, Foelker CE, Filho ENK, Souza FF, Bordalo-Rodrigues $\mathrm{M}$. Evaluation of ulnar variance on wrist MR imaging: is it a reliable measure? Skeletal Radiol 2020;49:723-30.

3. Ozer K, Zhu AF, Siljander B, Lawton JN, Waljee JF. The Effect of Axial Loading on Ulnar Variance. J Wrist Surg 2018;7:247-52.

4. Strike SA, Puhaindran ME. Tumors of the Hand and the Wrist. JBJS Rev 2020;8:e0141.

5. Meena S, Gupta A. Dorsal wrist ganglion: Current review of literature. J Clin Orthop Trauma 2014;5:59-64.

6. Turan A, Kose O, Aktan C, Unal M, Acar B, Sindel M. Radiographic analysis of anatomic risk factors for scaphoid fractures; A case-control study. Clin Imaging 2018;51:341-6.

7. Yoshioka H, Tanaka T, Ueno T, et al. Study of ulnar variance with high-resolution MRI: correlation with triangular fibrocartilage complex and cartilage of ulnar side of wrist. J Magn Reson Imaging 2007;26:714-9.

8. Roh YH, Kim S, Gong HS, Baek GH. Prevalence and clinical characteristics of radiographic central triangular fibrocartilage complex tears in symptomatic and asymptomatic individuals younger than 50 years. Arch Orthop Trauma Surg 2018;138:1173-8.

9. Roulet S, Gubbiotti L, Lakhal W, et al. Ulna shortening osteotomy for ulnar impaction syndrome: Impact of distal radioulnar joint morphology on clinical outcome. Orthop Traumatol Surg Res 2021;107:102970.

10. Ghalimah BA, Mimish RL, Khashoggi KG, Hamdi AS. A preliminary exploration of ulnar variance in healthy wrists at a tertiary hospital in Jeddah. Saudi Med J 2016;37:843-6.

11. Goldfarb CA, Strauss NL, Wall LB, Calfee RP. Defining ulnar variance in the adolescent wrist: measurement technique and interobserver reliability. J Hand Surg Am 2011;36:272-7.

12. Zhang A, Falkowski $A L$, Jacobson JA, Kim SM, Koh SH, Gaetke-Udager K. Sonography of Wrist Ganglion Cysts: Which Location Is Most Common? J Ultrasound Med 2019;38:2155-60.

13. Lowden CM, Attiah M, Garvin G, Macdermid JC, Osman S, Faber KJ. The Prevalence of Wrist Ganglia in an Asymptomatic Population: Magnetic Resonance Evaluation. J Hand Surg $\mathrm{Br}$ 2005;30:302-6.

14. Kuliński S, Gutkowska O, Mizia S, Martynkiewicz J, Gosk J. Dorsal and volar wrist ganglions: The results of surgical treatment. Adv Clin Exp Med 2019;28:95-102.

15. d'Ailly PN, Koopman JE, Selles CA, Rahimtoola ZO, Schep NWL. Patient-Related Outcomes of Arthroscopic Resection of Ganglion Cysts of the Wrist. J Wrist Surg 2021;10:31-5.

16. Bracken J, Bartlett M. Ganglion cysts in the paediatric wrist: magnetic resonance imaging findings. Pediatr Radiol 2013;43:1622-8. 